\title{
The Hyper-Kamiokande Project
}

\author{
Francesca Di Lodovico*i \\ Queen Mary University of London \\ E-mail: f.r.di.lodovico@gmul.ac.uk
}

The Hyper-Kamiokande experiment is a proposed next generation underground water Cherenkov detector. It will serve as the far detector of a long baseline neutrino oscillation experiment envisioned for the upgraded J-PARC beam. It is capable of observing - far beyond the sensitivity of the Super-Kamiokande detector - proton decays, atmospheric neutrinos, and neutrinos from astronomical origins. The baseline design of Hyper-Kamiokande is based on the highly successful Super-Kamiokande, but with a 20 times larger detector and higher beam power. With a total exposure of $7.5 \mathrm{MW} \times 10^{7} \mathrm{sec}$ integrated proton beam power (corresponding to $1.56 \times 10^{22}$ protons on target with a $30 \mathrm{GeV}$ proton beam) to a 2.5 -degree off-axis neutrino beam, it is expected that the leptonic $C P$ phase $\delta_{C P}$ can be determined to better than 19 degrees for all possible values of $\delta_{C P}$, and $C P$ violation can be established with a statistical significance of more than $3 \sigma(5 \sigma)$ for $76 \%$ (58\%) of the $\delta_{C P}$ parameter space. Using both $v_{e}$ appearance and $v_{\mu}$ disappearance data, the expected $1 \sigma$ uncertainty of $\sin ^{2} \theta_{23}$ is $0.015(0.006)$ for $\sin ^{2} \theta_{23}=0.5(0.45)$.

16th International Workshop on Neutrino Factories and Future Neutrino Beam Facilities - NUFACT2014, 25 -30 August, 2014

University of Glasgow, United Kingdom

\footnotetext{
* Speaker.

${ }^{\dagger}$ Partially supported by the grant ERC-207282.
} 


\section{Introduction}

The Hyper-Kamiokande (Hyper-K) experiment (see Refs. [1], [2], [3]) is the next generation flagship experiment for the study of neutrino oscillations, nucleon decays, and astrophysical neutrinos. The detector is a third generation underground water Cherenkov (WC) detector situated in Kamioka, Japan. It consists of a 1 million tonne water target which is about 20 times larger than that of the existing Super-Kamiokande (Super-K) detector. It will serve as the far detector for a long baseline neutrino oscillation experiment planned for the upgraded J-PARC beam. It will also serve as a detector capable of observing proton decays, atmospheric neutrinos, and neutrinos from astronomical origins enabling measurements that far exceed the current world best measurements. In the following sections, we first describe the expected neutrino landscape in the 2020s, then a description of the accelerator complex, the near and far detectors will be given, finally the physics potential of the experiment will be described with particular focus on the sensitivity to CP violation.

\section{Expected Neutrino Landscape in the 2020s}

The physics landscape in the 2020s will be shaped by the current and near future experiments, that will be able to advance our knowledge of neutrino physics from accelerator, reactor and cosmological observations. This will lead to a much improved understand of the neutrino oscillation parameters, including a possible measurement of the mass hierarchy. Several experiments will also be able to provide a more deailed measurement of neutrino masses, and possibly discover the nature of neutrinos, i.e. whether Majorana or Dirac, by measuring the neutrinoless double beta process. Following this expected progress, we will need a new experiment dedicated to the measurement of the leptonic CP violation. Moreover, this experiment would be able to further improve the neutrino oscillation parameters precision and to measure the mass hierarchy if not already achieved.

The Hyper-Kamiokande experiment will be in an excellent position to achieve this goal thanks to its important characteristics: very high statistics of neutrino events thanks to its large mass and a high beam power from the J-PARC neutrino beam, systematics understood from its near detectors, designed on purpose, and its previous experiments T2K and Super-Kamiokande. Its physics portafolio will also include the most precise sensitivity to proton decay and neutrinos from astrophysical sources.

\section{Experimental Setup}

\subsection{J-PARC}

J-PARC (Japan Proton Accelerator Research Complex) [4] is an accelerator research complex currently providing the beam to several experiments, among which the T2K experiment. It provides a narrow-band off-axis beam configuration at $2.5^{\circ}$.

The Hyper-Kamiokande experimment will utilize the full potential of this existing facility with future increase of the beam power to the design value of $750 \mathrm{~kW}$ and beyond.

The J-PARC accelerator complex consists of a normal-conducting LINAC as an injection system, a Rapid Cycling Synchrotron (RCS), and a Main Ring synchrotron (MR). In the fast extraction mode operation, the MR has achieved $1.24 \times 10^{14}$ protons per pulse (ppp) beam intensity, which is a 
world record for extracted ppp for any synchrotron. The corresponding beam power is $240 \mathrm{~kW}$. The plan for the upgrade to reach the design power of $750 \mathrm{~kW}$ is currently under way. This will double the current repetition rate by (i) replacing the magnet power supplies, (ii) replacing the RF system, and (iii) upgrading injection/extraction devices. The design power of $750 \mathrm{~kW}$ will be achieved well before Hyper-K will start data taking. Furthermore, conceptual studies on how to realize 1 2 MW beam powers and even beyond are now underway, such as by raising the RCS top energy, enlarging the MR aperture, or inserting an "emittance-damping" ring between the RCS and MR.

\subsection{Near Detectors}

The neutrino flux and cross-section models can be constrained by data collected at near detectors, situated close enough to the neutrino production point so that oscillation effects are negligible. The near detector data addresses important uncertainties in the neutrino flux or cross-section modeling.

The conceptual design of the near detectors is being developed based on the physics sensitivity studies outlined later in this document. In this section, basic considerations on the near detector requirements and conceptual designs are presented. More concrete requirements and detector design will be reported in future publications.

There are ongoing discussions on using the T2K near detectors [1], INGRID and ND280, possibly with an upgrade, for Hyper-Kamiokande . The T2K collaboration is in the process of discussing various upgrade possibilities at the ND280 site [5]. These include the deployment of heavy water $\left(\mathrm{D}_{2} \mathrm{O}\right)$ within the passive water targets in FGD2 that would allow the extraction of neutrino interaction properties on the quasi-free neutron in deuterium via a subtraction with data taken with light water $\mathrm{H}_{2} \mathrm{O}$. The use of a water-based liquid scintillator (WbLS) developed at BNL [6] is being explored in the context of a tracking detector with comparable or finer granularity than the FGD to allow the detailed reconstruction of hadronic system emerging from the neutrino interactions or a larger detector with coarser segmentation that would allow high statistics studies. Either would significantly enhance the study of neutrino interactions on water by reducing the reliance on subtraction and enhancing the reconstruction capabilities relative to the currently deployed passive targets. Finally, a high pressure TPC that can contain various noble gases ( $\mathrm{He}, \mathrm{Ne}, \mathrm{Ar}$ ) to serve both as the target and tracking medium is being studied. Such a detector would allow the ultimate resolution of the particles emitted from the target nucleus while allowing a study of the $A$-dependence of the cross-sections and final state interactions to rigorously test models employed in neutrino event generators.

Since many of the uncertainties on the modeling of neutrino interactions arise from uncertainties on nuclear effects, the ideal near detector should include the same nuclear targets as the far detector. In T2K near detectors, the P0D [7] and FGD [8] detectors include passive water layers, however extracting water only cross sections requires complicated analyses that subtract out the interactions on other materials in the detectors. An alternative approach is to build a water Cherenkov (WC) near detector to measure the cross section on $\mathrm{H}_{2} \mathrm{O}$ directly and with no need for a subtraction analysis.

Two conceptual designs for possible intermediate WC detectors have been studied. The Tokai Intermediate Tank for Unoscillated Spectrum (TITUS) is a 2 kiloton WC detector located about $2 \mathrm{~km}$ from the target at the same off-axis angle as the far detector. At this baseline the detector sees 
fluxes for the neutral current and $v_{e}$ backgrounds that are nearly identical to the Hyper-K fluxes. The detector geometry and the presence of a muon range detector are optimized to detect the high momentum tail of the muon spectrum. The use of Gd in TITUS allows to separate neutrino and antineutrino interactions. The $v$ PRISM detector is located $1 \mathrm{~km}$ from the target and is $50 \mathrm{~m}$ tall, covering a range of off-axis angles from 1-4 degrees, exploiting the corresponding spectra to better probe the relationship between the incident neutrino energy and final state lepton kinematics, a part of the interaction model with larger uncertainties arising from nuclear effects.

\subsection{The Hyper-Kamiokande Detector}

The Hyper-Kamiokande detector is a water Cherenkov detector in Kamioka with a total (fiducial) mass of 0.99 (0.56) million metric tons, approximately 20 (25) times larger than that of SuperKamiokande. Table 1 summarizes the baseline design parameters of the Hyper-K detector.

Table 1: Parameters of the Hyper-Kamiokande baseline design.

\begin{tabular}{lll}
\hline \hline Detector type & & Ring-imaging water Cherenkov detector \\
\hline Candidate site & Address & Tochibora mine \\
& & Kamioka town, Gifu, JAPAN \\
& Lat. & $36^{\circ} 21^{\prime} 20.105^{\prime \prime} \mathrm{N}^{\dagger}$ \\
& Long. & $137^{\circ} 18^{\prime} 49.137^{\prime \prime} \mathrm{E}^{\dagger}$ \\
& Alt. & $508 \mathrm{~m}$ \\
& Overburden & $648 \mathrm{~m}$ rock $(1,750 \mathrm{~m}$ water equivalent) \\
& Cosmic Ray Muon flux & $\sim 8 \times 10^{-7} \mathrm{sec}^{-1} \mathrm{~cm}^{-2}$ \\
& Off-axis angle for the J-PARC $v$ & $2.5^{\circ}$ (same as Super-Kamiokande) \\
& Distance from the J-PARC & $295 \mathrm{~km}(\mathrm{same}$ as Super-Kamiokande) \\
\hline Detector geometry & Total Water Mass & $0.99 \mathrm{Megaton}$ \\
& Inner Detector (Fiducial) Mass & $0.74(0.56)$ Megaton \\
& Outer Detector Mass & $0.2 \mathrm{Megaton}$ \\
\hline Photo-multiplier Tubes & Inner detector & 99,00020 -inch $\phi$ PMTs \\
& & $20 \%$ photo-coverage \\
& Outer detector & 25,0008 -inch $\phi$ PMTs \\
\hline Water quality & light attenuation length & $>100 \mathrm{~m} @ 400 \mathrm{~nm}$ \\
& Rn concentration & $<1 \mathrm{mBq} / \mathrm{m}^{3}$ \\
\hline \hline
\end{tabular}

${ }^{\dagger}$ World geographical coordination system

The candidate site for Hyper-K detector, located $8 \mathrm{~km}$ south of Super-K, is in the Tochibora mine. The J-PARC neutrino beamline is designed so that the existing Super-Kamiokande detector and the Hyper-K candidate site in the Tochibora mine have the same off-axis angle. The Mozumi mine under Mt. Ikeno-yama, where the Super-K detector is located, is another candidate site which can provide more overburden $(\geq 700 \mathrm{~m})$ than the Tochibora site $(\sim 650 \mathrm{~m})$ and reduced background levels for low-energy physics, such as solar neutrinos and supernova relic neutrinos. The geological 
surveys have been carried out at a vicinity of the candidate site, and detailed stability analyses of the cavern construction and evaluation of the construction period and cost are in progress.

For the baseline design, the Hyper-K detector is composed of two separated caverns as shown in Fig. 1 (left), each having an egg-shape cross section 48 meters wide, 54 meters tall, and 250 meters long as shown in Fig. 1 (right). The welded polyethylene tanks are filled up to a depth of $48 \mathrm{~m}$ with ultra-pure water: the total water mass equals 0.99 million tons.
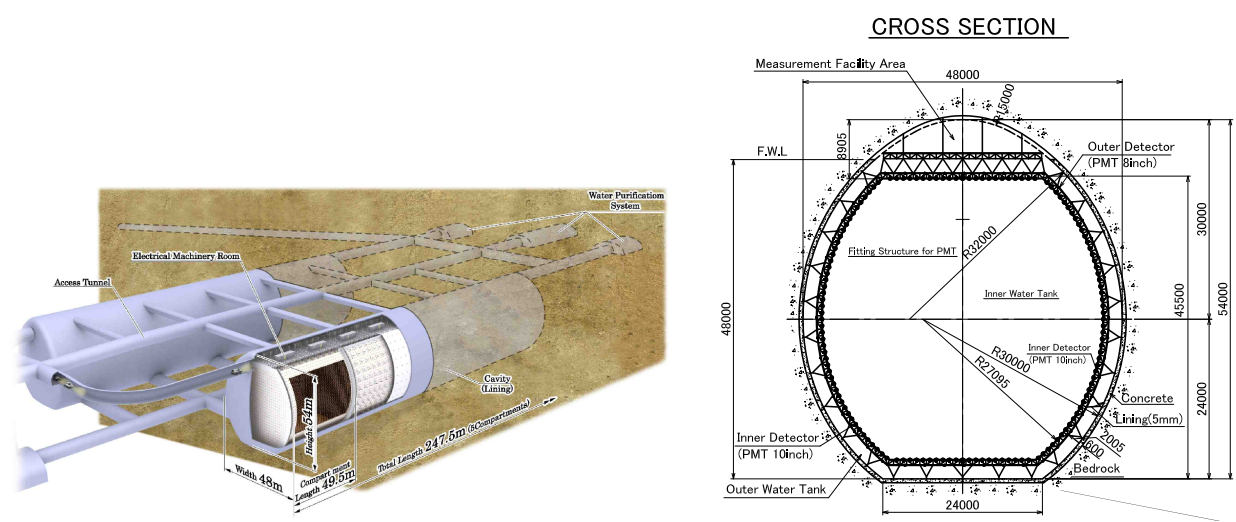

Figure 1: Left: schematic view of the Hyper-Kamiokande detector. Right: cross section view of the HyperKamiokande detector.

Table 2: The expected number of $v_{e}$ and $v_{\mu}$ candidate events for the appearance and disappearance final states, respectively. Normal Hierarchy (NH), $\sin ^{2} 2 \theta_{13}=0.1$ and $\delta_{C P}=0$ are assumed. The background is categorized by the flavor before oscillation.

\begin{tabular}{|c|c|c|c|c|c|c|c|}
\hline \multicolumn{8}{|c|}{ Appearance } \\
\hline & \multicolumn{2}{|c|}{ signal } & \multicolumn{4}{|c|}{ BG } & \multirow[t]{2}{*}{ total } \\
\hline & $v_{\mu} \rightarrow v_{e}$ & $\bar{v}_{\mu} \rightarrow \bar{v}_{e}$ & $v_{\mu}$ & $\bar{v}_{\mu}$ & $v_{e}$ & $\mathrm{NC}$ & \\
\hline$v$ mode & 3016 & 28 & 11 & 0 & 503 & $20 \quad 172$ & 3750 \\
\hline $\bar{v}$ mode & 396 & 2110 & 4 & 5 & 222 & $265 \quad 265$ & 3397 \\
\hline \multicolumn{8}{|c|}{ Disappearance } \\
\hline & \multicolumn{2}{|c|}{ signal } & \multicolumn{4}{|c|}{ BG } & \multirow[t]{2}{*}{ total } \\
\hline & $v_{\mu} \rightarrow v_{\mu}$ & $\bar{v}_{\mu} \rightarrow \bar{v}_{\mu}$ & $v_{e}$ & $\bar{v}_{e}$ & $\mathrm{NC}$ & $v_{\mu} \rightarrow v_{e}$ & \\
\hline$v$ mode & 17225 & 1088 & 11 & 1 & 999 & 49 & 19372 \\
\hline $\bar{v}$ mode & 10066 & 15597 & 7 & 7 & 1281 & 6 & 26964 \\
\hline
\end{tabular}

Each tank will be optically separated by segmentation walls located every $49.5 \mathrm{~m}$ to form 5 (in total 10) compartments, such that event triggering and event reconstruction can be performed in each compartment separately and independently. Because the compartment dimension of $50 \mathrm{~m}$ is comparable with that of Super- $\mathrm{K}(36 \mathrm{~m})$ and is shorter than the typical light attenuation length in water achieved by the Super-K water filtration system ( $>100 \mathrm{~m} @ 400 \mathrm{~nm}$ ), we expect that the 
detector performance of Hyper-K will be basically the same as that of Super-K. The water in each compartment is further optically separated into three regions. The inner region has a barrel shape of $42 \mathrm{~m}$ in height and width, and $48.5 \mathrm{~m}$ in length, and is viewed by an inward-facing array of 20-inch diameter photomultiplier tubes (PMTs). The entire array consists of 99,000 Hamamatsu R3600 PMTs, uniformly surrounding the region and giving a photocathode coverage of $20 \%$. The PMT type, size, and number density are subject to optimization, currently underway in the eGADS detector (see e.g. Ref. [9]) in the Kamioka mine.

An outer region completely surrounds the 5 (in total 10) inner regions and is equipped with 25,000 8-inch diameter PMTs. This region is $2 \mathrm{~m}$ thick at the top, bottom, and barrel sides, except at both ends of each cavern, where the outer region is larger than $2 \mathrm{~m}$ due to rock engineering considerations. A primary function of the outer detector is to reject entering cosmic-ray muon backgrounds and help to identify nucleon decays and neutrino interactions occurring in the inner detector.

There are several efforts to improve the electronics and DAQ SK-baseline design to match the needs of a much larger detector. Similarly, the calibration system needs to be as automated as possible to be able to calibration of all the compartments.

\section{Physics Potential}

A recent update of the previous sensitivity studies presented in 2011 [1] is based on the latest value of $\theta_{13}$ (which was not known at that time) and more generally the latest knowledge of the oscillation parameters. It uses a framework for the oscillation analysis developed by $\mathrm{T} 2 \mathrm{~K}$ and reported in [10], and the latest systematic errors based on the experience and prospects of the T2K experiment. The document, that contains an update on the R\&D of the experiment as well was submitted in April 2014 to the J-PARC Programme Advisory Committee (PAC) [2] and a more recent version focussing on physics only was submitted for publication [3]. An integrated beam power of $7.5 \mathrm{MW} \times 10^{7} \mathrm{sec}$ is assumed in this study. It corresponds to $1.56 \times 10^{22}$ protons on target (POT) with a $30 \mathrm{GeV} \mathrm{J-PARC} \mathrm{beam.} \mathrm{The} \mathrm{ratio} \mathrm{of} \mathrm{neutrino} \mathrm{and} \mathrm{anti-neutrino} \mathrm{running} \mathrm{time} \mathrm{is}$ assumed to be $1: 3$. The oscillation parameters used for the sensitivity analysis are: $\sin ^{2} 2 \theta_{13}(0.1$, fitted), $\delta_{C P}\left(0\right.$, fitted), $\sin ^{2} \theta_{23}$ (0.5, fitted), $\Delta m_{32}^{2}\left(2.4 \times 10^{-3} \mathrm{eV}^{2}\right.$, fitted), mass hierarchy (normal, fitted), $\sin ^{2} 2 \theta_{12}\left(0.8704\right.$, fixed) and $\Delta m_{12}^{2}\left(7.6 \times 10^{-5} \mathrm{eV}^{2}\right.$, fixed), where in parenthesis the nominal values used in the fits and the treatment used in the fits are indicated. The criteria to select $v_{e}$ and $v_{\mu}$ candidate events are based on those developed for and established with the Super-K and T2K experiments, and the corresponding number of expected events is shown is Table 2.

Figure 2, upper row, shows the expected significance to exclude $\sin \delta_{C P}=0$ (the $\mathrm{CP}$ conserved case), for the normal (left) and inverted (right) mass hierarchy. The significance is calculated as $\sqrt{\Delta \chi^{2}}$, where $\Delta \chi^{2}$ is the difference of $\chi^{2}$ between the trial value of $\delta_{C P}$ and either $\delta_{C P}=0^{\circ}$ or $180^{\circ}$ (the smaller value of the difference is taken). We have also studied the case with a reactor constraint [11], but the result changes only slightly. Figure 2 (bottom row, left) shows the fraction of $\delta_{C P}$ for which $\sin \delta_{C P}=0$ is excluded with $3 \sigma$ and $5 \sigma$ of significance as a function of the integrated beam power. The normal mass hierarchy is assumed. The results for the inverted hierarchy is almost the same. $C P$ violation in the lepton sector can be observed with 3(5) $\sigma$ significance for 76(58)\% of the possible values of $\delta_{C P}$. Figure 2 (bottom row, right) shows the $1 \sigma$ uncertainty of $\delta_{C P}$ as a 

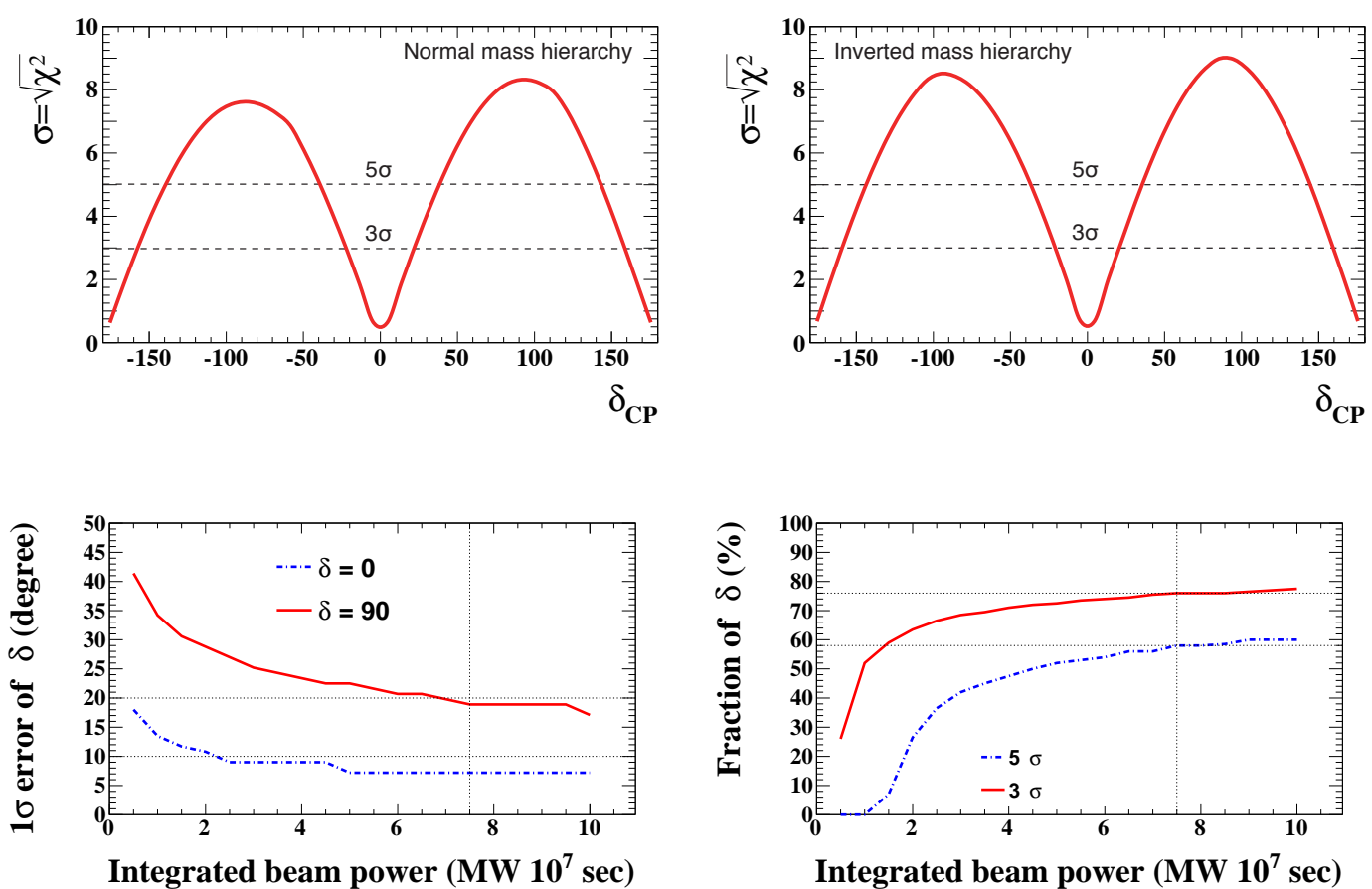

Figure 2: Upper row: expected significance to exclude $\sin \delta_{C P}=0$. Left: normal hierarchy case. Right: inverted hierarchy case. Bottom row: fraction of $\delta_{C P}$ for which $\sin \delta_{C P}=0$ can be excluded with $3 \sigma$ (red solid line) and $5 \sigma$ (blue dashed line) significance as a function of the integrated beam power (NH). Right plot: expected $1 \sigma$ uncertainty of $\delta_{C P}$ as a function of integrated beam power.

function of the integrated beam power. With $7.5 \mathrm{MW} \times 10^{7} \mathrm{sec}$ of exposure $\left(1.56 \times 10^{22} \mathrm{POT}\right)$, the value of $\delta_{C P}$ can be determined to better than $19^{\circ}$ for all values of $\delta_{C P}$. The use of $v_{\mu}$ sample in addition to $v_{e}$ enables us to also measure $\sin ^{2} \theta_{23}$ and $\Delta m_{32}^{2}$ and Hyper-K will be able to provide a precise measurement of $\sin ^{2} \theta_{23}$ and $\Delta m_{32}^{2}$. Figure 3 (left) shows the $90 \% \mathrm{CL}$ allowed regions on the $\sin ^{2} \theta_{23}-\Delta m_{32}^{2}$ plane, for the true values of $\sin ^{2} \theta_{23}=0.45$ and $\Delta m_{32}^{2}=2.4 \times 10^{-3} \mathrm{eV}^{2}$. With a constraint on $\sin ^{2} 2 \theta_{13}$ from the reactor experiments (right), the octant degeneracy is resolved and $\theta_{23}$ can be precisely measured.
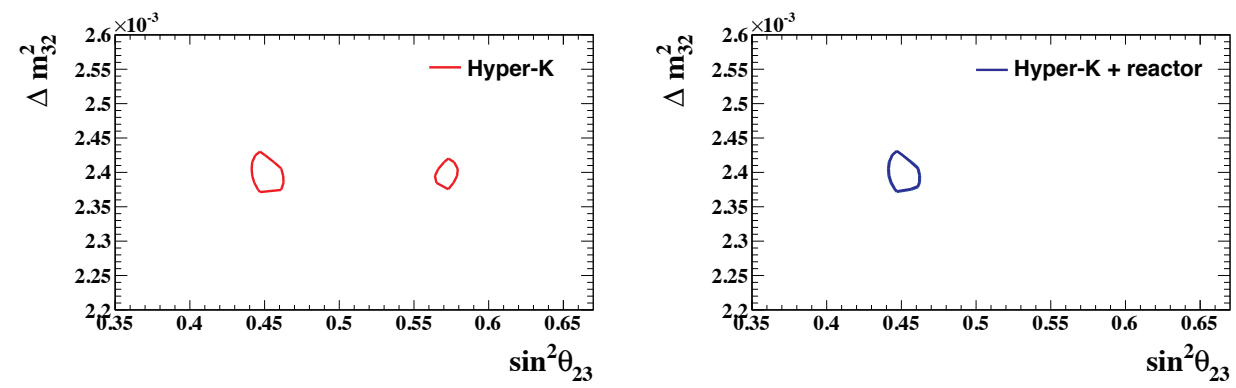

Figure 3: $90 \% \mathrm{CL}$ allowed regions in the $\sin ^{2} \theta_{23}-\Delta m_{32}^{2}$ plane. The true values are $\sin ^{2} \theta_{23}=0.45$ and $\Delta m_{32}^{2}=2.4 \times 10^{-3} \mathrm{eV}^{2}$. The effect of the systematic uncertainties is included. Left plot: Hyper-K only. Right plot: With reactor constraint.

\section{Atmospheric Neutrinos}


Atmospheric neutrinos can provide independent and complementary information to the accelerator beam program on the study of neutrino oscillation. Assuming a 10 year exposure, Hyper-K's sensitivity to the mass hierarchy and the octant of $\theta_{23}$ by atmospheric neutrino data are shown in Figure 4, left and right figures, respectively. Depending upon the true value of $\theta_{23}$ the sensitivity changes considerably, but for all currently allowed values of this parameter the mass hierarchy sensitivity exceeds $3 \sigma$ independent of the assumed hierarchy. If $\theta_{23}$ is non-maximal, the atmospheric neutrino data can be used to discriminate the octant at $3 \sigma$ if $\sin ^{2} 2 \theta_{23}<0.99$.
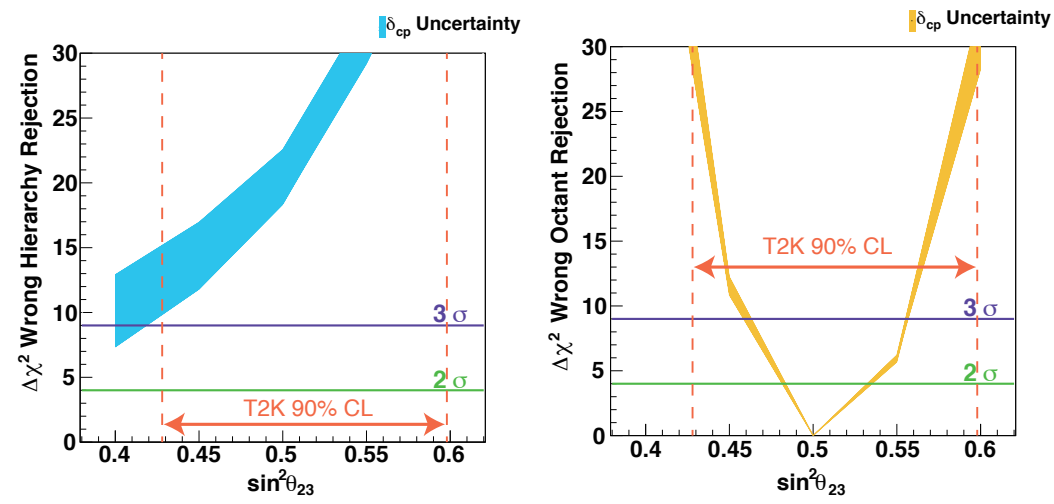

Figure 4: Atmospheric neutrino sensitivities for a ten year exposure of Hyper-K assuming the mass hierarchy is normal. Left: the $\Delta \chi^{2}$ discrimination of the wrong hierarchy hypothesis as a function of the assumed true value of $\sin ^{2} \theta_{23}$. Right: the discrimination between the wrong octant for each value of $\sin ^{2} \theta_{23}$. The uncertainty from $\delta_{c p}$ is given by the thickness of the band.

\section{Proton Decay Search}

Large water Cherenkov detectors have excellent sensitivities for nucleon decays. for more than a decade Super-Kamiokande has had the world's best limits, generally by an order of magnitude or more, on most of the current theoretically favored decay modes. Among many possible nucleon decay modes $p \rightarrow e^{+} \pi^{0}$ and $p \rightarrow \bar{v} K^{+}$have been the subjects of the most intense interest. If the proton lifetime is shorter than $5.7 \times 10^{34}$ years for the $p \rightarrow e^{+} \pi^{0}$ mode, or shorter than $1.0 \times 10^{34}$ years for $p \rightarrow \bar{v} K^{+}$, we could discover a signal over the atmospheric neutrino background events with a $3 \sigma$ significance by collecting a 5.6 Megaton-year exposure. The sensitivity of Hyper-Kamiokande can cover most of the predicted range of the major GUTs models. Fig. 5 (left) shows the sensitivity of Super-Kamiokande and Hyper-Kamiokande as a function of the year. The nucleon decay results from Hyper-Kamiokande can supercede the ones from Super-Kamiokande within a single year of running. Moreover, many other nucleon decay searches in Hyper-Kamiokande have been studied.

\section{Supernovas}

Since neutrinos interact weakly with matter, almost $99 \%$ of the released energy from an exploding star is carried by neutrinos. As a result, the detection of supernova neutrinos gives direct information of energy flow during the explosion. Moreover, the supernova neutrinos also give us an opportunity to investigate unknown properties of neutrinos, e.g. the neutrino mass hierarchy using the time variation of the energy spectrum, the neutrino direct mass studying the energy dependence of arrival times at the onset of the burst, their lifetime from neutrinos studies from distance sources, etc. Figure 5 (right) shows the expected number of supernova neutrino events at Hyper-K versus the 

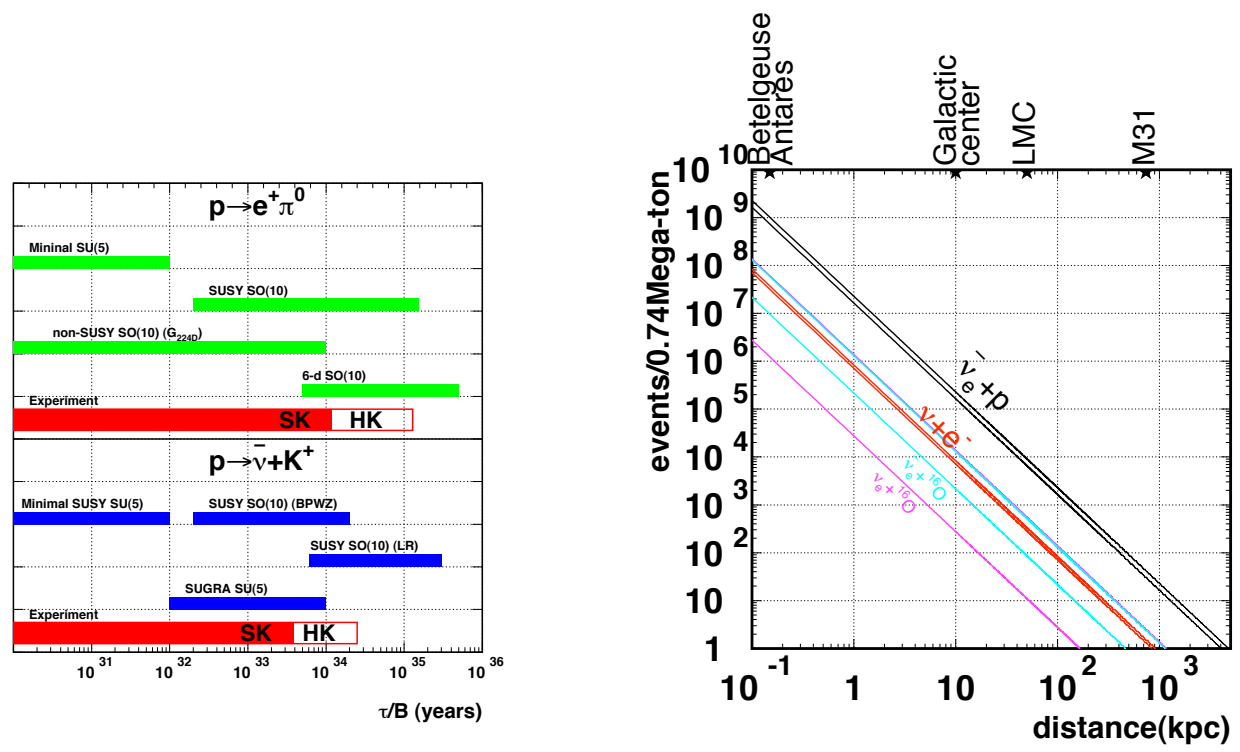

Figure 5: Left plot: Proton lifetime predictions of several GUT models, the current experimental limits $(90 \%$ CL) by Super-K, and the sensitivities of Hyper-Kamiokande with a 5.6 Megaton·year exposure. HyperKamiokande can cover most of the predicted range of the leading GUT models. Reght plot: Expected number of supernova burst events for each interaction as a function of the distance to a supernova. The band of each line shows the possible variation due to the assumption of neutrino oscillations.

distance to a supernova. At the Hyper-K detector, we expect to see about 165,000 230,000 inverse beta events, 7,000 8,000 $v_{e}$-scattering events, 300 14,000 $v_{e}^{16} \mathrm{O}$ CC events, and 2,000 13,000 $\bar{v}_{e}^{16} \mathrm{O} C \mathrm{C}$ events, in total 170,000 260,000 events, for a $10 \mathrm{kpc}$ supernova. Moerover, the neutrinos produced by all the supernova explosions since the beginning of the universe are called supernova relic neutrinos (SRN), and should fill the present universe. Their flux is estimated to be a few tens $/ \mathrm{cm}^{2} / \mathrm{sec}$. By detecting these neutrinos it is possible to explore the history of how heavy elements have been synthesized since the onset of stellar formation. In order to reduce background, lower the energy threshold, individually identify true inverse beta events by tagging their neutrons, and thereby positively detect SRN signals, an addition of about $0.1 \%$ gadolinium to the tank is proposed, based on Ref. [12] for Super-K. But, in order to measure the spectrum of the SRN and analyze the history of the universe, we need Hyper-K, a megaton-scale detector.

\section{Further Measurements}

Hyper-Kamiokande will be able to perform several other high sensitivity measurements and searches:

- Solar Neutrinos: assuming sufficient depth to overcome cosmic-muon-induced spallation background, Hyper-K will observe $\sim 115,000$ elastic solar ${ }^{8} \mathrm{~B}$ neutrino-electron scatters per year. Hyper-K will be very sensitive to the solar day/night effect, determine the solar zenith angle variation amplitude to $\sim 0.5 \%$ within 5 years, and measure $\Delta m_{21}^{2}$ with a precision comparable to that of current reactor antineutrino experiments.

- Indirect Dark Matter Search: in indirect searches, signals are from neutrinos emitted by WIMP annihilations which occur in the Sun, Earth, or in the halo, where WIMPs are trapped by gravitational potentials. Hyper-K can improve the present sensitivity significantly for 
lighter WIMPs $\leq 100 \mathrm{GeV}$.

- Other Astrophysical Seaches: Other astronomical neutrino searches such as GRB neutrinos, galactic diffuse neutrinos, etc. can be also performed in Hyper-K with its large acceptance in a wide energy range.

- Geophysical Neutrinos: Hyper-Kamiokande's unique capability to detect a large number of atmospheric neutrinos of around $\mathrm{GeV}$ energies enables us to perform the neutrino oscillation radiography using the Mikheyev Smirnov Wolfenstein (MSW) effect. Hyper-K would be able to perform the neutrino oscillation radiography for surveying the internal structure of the Earth with considerably higher statistics than any other experiment.

\section{Conclusions}

Hyper-Kamiokande is the next generation long baseline neutrino experiment in Japan. It will be able to measure $\delta_{C P}$ with 3(5) $\sigma$ significance for $76(58) \%$ of the possible values of $\delta_{C P}$ to better than $19^{\circ}$ for all values of $\delta_{C P}$. Complementing the beam results with the atmospheric neutrinos it will also be able to measure the mass hierarchy and the octant of $\theta_{23}$. Moreover, it is capable of observing, far beyond the sensitivity of the current experiments, proton decays, atmospheric neutrinos, and neutrinos from astrophysical origins.

\section{References}

[1] K. Abe, T. Abe, H. Aihara, Y. Fukuda, Y. Hayato, et al. Letter of Intent: The Hyper-Kamiokande Experiment — Detector Design and Physics Potential —. arXiv:1109.3262 [hep-ex] (2011).

[2] Hyper-Kamiokande Working Group, :, K. Abe, H. Aihara, C. Andreopoulos, et al. A Long Baseline Neutrino Oscillation Experiment Using J-PARC Neutrino Beam and Hyper-Kamiokande. arXiv:1412.4673 [physics.ins-det] (2014).

[3] K. Abe et al. Physics Potential of a Long Baseline Neutrino Oscillation Experiment Using J-PARC Neutrino Beam and Hyper-Kamiokande. arXiv:1502.05199 [hep-ex] (2015).

[4] M. Ikegami Y. Irie T. Kato Y. Yamazaki, K. Hasegawa et al. Accelerator technical design report for J-PARC. KEK-REPORT-2002-13, JAERI-TECH-2003-044, J-PARC-03-01 (2003).

[5] K. Abe and others (T2K Collaboration). Physics Potential and Sensitivities of T2KAccelerator technical design report for J-PARC. T2K Report to 17th J-PARC PAC, Sep. 2013.

[6] M. Yeh, S. Hans, W. Beriguete, R. Rosero, L. Hu, et al. A new water-based liquid scintillator and potential applications. Nucl.Instrum.Meth., A660:51-56, 2011.

[7] S. Assylbekov et al. The t2k nd280 off-axis pi-zero detector. Nucl. Instrum. Meth., A686:48-63, 2012.

[8] P.-A. Amaudruz et al. The t2k fine-grained detectors. Nucl. Instrum. Meth., A696:1-31, 2012.

[9] Andrew Renshaw. Research and Development for a Gadolinium Doped Water Cherenkov Detector. Phys.Procedia, 37:1249-1256, 2012.

[10] K. Abe et al. Neutrino Oscillation Physics Potential of the T2K Experiment. 2014.

[11] K.A. Olive et al. Review of particle physics. Chin.Phys., C38:090001, 2014.

[12] John F. Beacom and Mark R. Vagins. GADZOOKS! Anti-neutrino spectroscopy with large water Cherenkov detectors. Phys.Rev.Lett., 93:171101, 2004. 